\title{
CRESCIMENTO DA PLANTA E ABSORÇÃO DE N EM FUNÇÃO DA INOCULAÇÃO COM RIZÓBIO E ADUBAÇÃO MINERAL EM SOJA NODULANTE E NÃO-NODULANTE
}

\author{
Hipólito A. A. Mascarenhas ${ }^{1,3}$ \\ Takashi Muraoka 2,3 \\ Roberto T. Tanaka ${ }^{1,3}$ \\ Edimilson J. Ambrosano ${ }^{1,3}$ \\ Paulo C.O. Trivelin ${ }^{2,3}$
}

\section{RESUMO}

O experimento foi conduzido EM condições de casa de vegetação, em vasos coM 5,2 kg de solo, para verificar o efeito da adubação nitrogenada e da inoculação com Bradyrhizobium japonicum sobre genótipos de soja e isolinhas que nodulam e que não nodulam. Usou-se a amostra de latossolo vermelho amarelo fase arenosa, que teve sua saturação por bases elevada para $70 \%$ e foi também adubada com fósforo e potássio. $O$ delineamento experimental utilizado foi o inteiramente casualizado, em esquema fatorial, (seis tratamentos, três épocas de colheita das plantas), com quatro repetições. Os tratamentos testados foram: 1) IAC-14 (nodulante) inoculado; 2) IAC-15 (nodulante) inoculado; 3) isolinha D.71.9230 (nodulante) inoculado 4) isolinha D.71.9231 (nãonodulante) inoculado; 5) isolinha D71.9231 (não nodulante) não inoculado; 6) isolinha D71.9231 (não-nodulante) não inoculado + $100 \mathrm{~kg} / \mathrm{ha}$ de N. Foram cultivadas quatro plantas por vaso. As plantas foram colhidas

\footnotetext{
' Pesquisador Científico, Centro de Plantas Graníferas, IAC, C.P.28, 13.001970 Campinas, SP.E-mail hipolito@cec.iac.br

${ }^{2}$ Livre Docente, CENA,C.P. 96, 13418-260,Piracicaba, SP.

${ }^{3} \mathrm{Com}$ bolsa de produtividade em pesquisa de CNPq.
} 
aos 30, 48 e 56 dias após a emergência (DAE). Em todas elas foi determinada a massa seca da parte aérea, das raízes e a quantificação da fixação de $\mathrm{N}$, esta por meio da técnica de diluição isotópica com a marcação do solo com ${ }^{15} \mathrm{~N}$. Os resultados mostraram que a isolinha isogênica D71.9231 (não-nodulante), na época do florescimento (56 DAE), com ou sem inoculação, apresentou a menor massa seca de parte aérea ou raízes, porém, quando recebeu $100 \mathrm{~kg} / \mathrm{ha}$ de $\mathrm{N}$, igualou-se à das nodulantes que não receberam inoculante; isto indica a necessidade deste elemento quando não há simbiose. Observou-se também baixo teor de $\mathrm{N}$ total na isolinha D71.9231, mas, com aplicação de $100 \mathrm{~kg} / \mathrm{ha}$ de $\mathrm{N}$, o N total praticamente dobrou, mas foi ainda inferior ao dos nodulados. A maior quantidade de $\mathrm{N}$ foi retirada do solo pelo tratamento D71.9231 sem inoculante +100 $\mathrm{kg} / \mathrm{ha}(89 \%)$, sendo que os $11 \%$ restantes originaram-se do fertilizante. Já na linhagem nodulante, a proporção foi de $37 \%$ do solo e $63 \%$ da simbiose.

Palavras-chave: soja, isolinhas nodulantes e não-nodulantes, nitrogênio mineral, simbiose.

\section{ABSTRACT}

\section{PLANT GROWTH AND ABSORPTION OF N WITH THE USE OF RHYZOBIA AND NITROGEN FERTILIZER IN SOYBEAN NODULATED AND NON-NODULATED}

An experiment was carried out in a greenhouse in the Department of Legumes of the Centro de Graníferas (IAC) in Campinas, São Paulo, Brazil, in pots containing $5.2 \mathrm{~kg}$ of soil to verify the effect of nitrogen fertilization and inoculation using two soybean cultivars and two nodulating and non-nodulating isolines. The soil utilized was Red Yellow Latosol sandy phase. Dolomitic lime was added to increase the V\% value to 70 , nutrients $\mathrm{P}$ and $\mathrm{K}$ in the form of superphosphate and potassium chloride were utilized also. The experimental design was a factorial with six treatments $\mathrm{x}$ three dates of harvest, with four replications. The 
treatments were: 1) IAC-14 (nodulated) inoculated. 2) IAC-15 (nodulated) inoculated. 3) isoline D71.9230 (nodulated) inoculated. 4 ) isoline D71.9231 (non-nodulated) inoculated. 5) isoline D71.9231 (nonnodulated) not inoculated. 6) isoline D71.9231 (non-nodulated) not inoculated $+100 \mathrm{~kg} / \mathrm{ha}$ of $\mathrm{N}$. Eight seeds were sown and the four most vigorous plants were kept in each pot. The times of harvesting was 30, 48 and 56 days after emergence (DAE). At each harvest dry matter weight of above ground parts and roots was determined. To determine the quantity of $\mathrm{N}$ fixed the dilution isotopic technique was utilized, tagging the soil with ${ }^{15} \mathrm{~N}$. The results show that isoline D71.9231 (non-nodulated) at flowering time with or without inoculation produced the least dry matter weight of the above ground parts and roots, where as when $100 \mathrm{~kg} / \mathrm{ha}$ of $\mathrm{N}$ was supplied the non-nodulating isoline produced dry matter weight similar to the nodulated cultivars showing that nitrogen should be supplied when no symbiosis occurs. Low concentration of total $\mathrm{N}$ was also observed in the isoline D71.9231; with the application of $100 \mathrm{~kg} /$ ha of $\mathrm{N}$ the total $\mathrm{N}$ practically doubled but yet the quantity was lower than that of nodulating cultivars. The greatest quantity of $\mathrm{N}$ was taken up from the soil by isoline D71.9231 without inoculant $+100 \mathrm{~kg} /$ ha of $\mathrm{N}(89 \%)$ and only $11 \%$ came from the fertilizer. In the nodulated cultivars an average of $37 \%$ of the $\mathrm{N}$ came from the soil, whereas $63 \%$ was fixed.

Key words: soybean cultivars, nodulating and non-nodulating isolines, mineral nitrogen, symbioses

\section{INTRODUÇÃO}

A fixação simbiótica de nitrogênio $(\mathrm{N})$ em soja deve necessariamente ser suplementada com $\mathrm{N}$ do solo e com fertilizante, para que a cultura atinja o máximo crescimento e produtividade (Allos \& Bartholomew, 1959; Harper, 1974; Norman, 1944; Norman \& Krampitz, 1945), Existe muita controvérsia sobre a validade da suplementação de $N$ do solo por meio de fertilizante, na presença da fixação simbiótica. A 
soja pode rapidamente utilizar tanto o $\mathrm{N}$ simbiótico quanto o disponível no solo (Allos \& Bartholomew,1959; Harper, 1974; Norman ,1944; Umbreit \& Fred, 1936; Weber, 1966b), mas a quantidade de N simbiótico fixado é inversamente proporcional à quantidade de $\mathrm{N}$ disponível (Allen \& Baldwin, 1954; Allos \& Bartholomew, 1959; Weber, 1966b). Quando suprida em quantidade que excedam às necessidades para crescimento da planta, o N pode impedir a fixação simbiótica (Allos \& Bartholomew, 1959, Weber, 1966a). A vantagem econômica do $\mathrm{N}$ fixado simbioticamente, tem sido constantemente observada (Mascarenhas et al., 1967; Hungria et al. 1998). Tentativas para usar efetivamente quantidades de fertilizantes nitrogenados sem inibir o processo simbiótico, têm geralmente falhado. Hinson (1974) relatou que respostas ocasionais a fertilizantes nitrogenados, em soja, não devem ser consideradas como evidências de que o $\mathrm{N}$ deva ser usado como regra geral. Os dados desse autor sugerem que as respostas não são frequentes, são imprevisíveis e geralmente não econômicas. Porém, Novo et al. (1997) demostraram que, no inverno, há resposta ao nitrogênio mineral, devido à baixa fixação de nitrogênio do ar. Mascarenhas et al. (1984) usando isolinhas que nodulam ou não nodulam mostraram que, na presença de inoculante, a linhagem D71.9230, nodulante, produziu aproximadamente dez vezes mais sementes que a linhagem não nodulante (D71.9231). Quando aplicados $50 \mathrm{~kg} / \mathrm{ha}$ de $\mathrm{N}$ em cobertura, as plantas que não nodulam produziram o dobro de sementes, quando comparadas ao tratamento sem nitrogênio. Contudo, não atingiram a produtividade da isolinha que nodula e apresentaram sintomas de dificiência de $\mathrm{N}$.

O objetivo deste trabalho foi determinar o efeito da adubação nitrogenada e quantificar as taxas de fixação de nitrogênio em isolinhas de soja nodulantes e não nodulantes, em condições de casa de vegetação.

\section{MATERIAL E MÉTODOS}

O experimento foi conduzido em casa de vegetação em vasos com $5,2 \mathrm{~kg}$ de um latossolo vermelho amarelo, fase arenosa, que apresentava 
as seguintes características químicas: $\mathrm{pH}\left(\mathrm{CaCl}_{2}\right) 5,2 ; \mathrm{M} . \mathrm{O} .13 \mathrm{~g} / \mathrm{dm}^{3} ; \mathrm{V}$ $59 \%$; P extraído com resina $3 \mu \mathrm{g} / \mathrm{cm}^{3} ; \mathrm{K} 0,3 \mathrm{mmol}_{\mathrm{c}} / \mathrm{dm}^{3}$; Ca $20 \mathrm{mmol} /$ $\mathrm{dm}^{3} ; \mathrm{Mg} 3 \mathrm{mmol} / \mathrm{dm}^{3}$. A correção da amostra do solo foi feita por meio de calagem para elevar a saturação por bases para $70 \%$ e a adubação com p e K foi fornecida na forma de superfosfato simples e cloreto de potássio, respectivamente. O delineamento experimental utilizado foi o inteiramente casualizado, em esquema fatorial (seis tratamentos $\mathrm{x}$ três épocas de colheita das plantas), com quatro repetições. Os tratamentos testados foram: 1) IAC-14 (nodulante) inoculado; 2) IAC-15 (nodulante) inoculado; 3) isolinha D71.9230 (nodulante) inoculado; 4) isolinha D.71.9231 (não-nodulante) inoculado; 5) isolinha D71.9231 (não-nodulante) não inoculado; 6) isolinha D71.9231 (não-nodulante) não inoculado + $100 \mathrm{~kg} / \mathrm{ha}$ de N. Após o desbaste foram deixadas quatro plantas/vaso. As plantas, colhidas aos 30, 48 e 56 dias após a emergência (DAE), foram secas em estufa a $60^{\circ} \mathrm{C}$ durante 3 dias. Em cada época foi determinada a massa seca da parte aérea e das raízes, e o teor de $\mathrm{N}$ total e ${ }^{15} \mathrm{~N}$ segundo Bremner \& Mulvaney, 1982 e Trivelin et al. 1973. O nitrogênio fixado na parte aérea foi calculado para as três épocas. Na quantificação da fixação de nitrogênio foi utilizada a técnica da diluição isotópica com a marcação do solo com ${ }^{15} \mathrm{~N}$ (Fried \& Middelboe, 1977). A determinação da fixação biológica foi feita com uma planta controle não-fixadora (isolinha D71.9331 não nodulante). $\mathrm{O} \mathrm{N}$ do solo, no qual as plantas foram cultivadas, foi marcado com ${ }^{15} \mathrm{~N}\left(10 \%\right.$ de átomos de $\left.{ }^{15} \mathrm{~N}\right)$. O N marcado corresponde a dose de 10 $\mathrm{mg} / \mathrm{kg}$ de $\mathrm{N}$, e foi aplicado na forma de solução em vaso.

Para o cálculo da porcentagem de $\mathrm{N}_{2}$ fixado foi usada a seguinte equação ( McAuliffe et al., 1958):

$\% \mathrm{~N}$ prov. da fixação $=\left(1-\frac{\text { Átomo } \%{ }^{15} \mathrm{~N} \text { exec. } \text { Leguminosas }}{\text { Átomo } \%{ }^{15} \mathrm{~N} \text { exec. planta controle }}\right) \times 100$.

A quantidade de $\mathrm{N}$ na planta de soja proveniente da fixação(Q fix) foi obtida pela equação: 


$$
\mathrm{Q} \text { fix }=\frac{\% \mathrm{~N} \text { fix }}{100} \times \mathrm{QNT}
$$

onde QNT = quantidade de $\mathrm{N}$ total na planta de soja.

$\mathrm{Na}$ determinação do nitrogênio da planta proveniente do fertilizante, adicionou-se o equivalente a $100 \mathrm{~kg}$ de $\mathrm{N} /$ ha na forma de ${ }^{15} \mathrm{~N}$ utilizando uréia com 10 átomos $\%$ de ${ }^{15} \mathrm{~N}$ em excesso.

Foram realizadas determinações de nitrogênio total, pelo método Semi Microkjeldahl (Bremner, 1965a) e de abundância isotópica (Bremner, 1965b, Trivelin et al., 1973). Foram calculadas a porcentagem do nitrogênio na planta proveniente do fertilizante (\%NPPF), a quantidade de nitrogênio na planta proveniente do fertilizante (QNPPFmg), e a porcentagem de recuperação pela planta do nitrogênio do fertilizante $(\mathrm{R} \%)$, por meio das equações 1 a 3 .

$$
\begin{aligned}
& \% \mathrm{NPPF}=(\mathrm{a} / \mathrm{b}) 100 \\
& \mathrm{QNPPF}=(\% \mathrm{NPPF} / 100) \mathrm{NT} \\
& \mathrm{R} \%=(\mathrm{QNPPF} / \mathrm{NF}) 100
\end{aligned}
$$

sendo a e b as abundâncias de ${ }^{15} \mathrm{~N}$ (átomos \% em excesso) na planta e no fertilizante respectivamente; NT o nitrogênio total na parte aérea (mg por vaso) e NF a dose de $\mathrm{N}$-fertilizante (mg por vaso).

\section{RESULTADOS E DISCUSSÃO}

Uma comparação horizontal dos dados da Tabela 1 evidencia o crescimento de forma quadrática das plantas em função das épocas de colheita, com exceção do crescimento da parte aérea do cultivar IAC-15 e das raízes da isolinha D71.9230 (nodulante), que se ajustaram de forma 
linear. Notou-se que a cada intervalo avaliado, a produção de matéria seca da parte aérea triplicou nos intervalos entre as duas primeiras épocas e duplicou entre a segunda e a terceira. Porém o aumento da matéria seca das raízes não seguiu a mesma taxa; observou-se aumento entre a primeira e a segunda época de colheita, de aproximadamente $80 \%$, e aumento bem menor da segunda para a terceira época (aproximadamente 11\%).

Aos $30 \mathrm{DAE}$, a produção de matéria seca de parte aérea dos tratamentos IAC-14 e IAC-15 foi significativamente inferior à dos demais tratamentos. Aos 48 dias, a massa de matéria seca dos tratamentos foi semelhante, não havendo diferenças significativas pelo teste de Duncan a $5 \%$ de probabilidade. Por outro lado, aos 56 DAE o tratamento D71.9231 sem inoculação $+100 \mathrm{~kg} / \mathrm{ha}$ não diferiu da linhagem isogênica nodulante D71.9230 e do cultivar IAC-15, mas foi superior ao cultivar IAC-14 e à linhagem isogênica não nodulante D71.92.31, inoculada ou não (Tabela 1). Na época do florescimento das plantas D71.9231 (com e sem inoculante), foram observadas folhas amarelas e sua queda prematura, um dos sintomas de deficiência de nitrogênio. O tratamento D.71.9231 (inoculado) $+100 \mathrm{~kg} / \mathrm{ha}$ de $\mathrm{N}$ também apresentou sintomas de deficiência de $\mathrm{N}$ nas folhas mas não tão acentuados como os dos tratamentos anteriores, e sem queda das folhas. As folhas das plantas dos genótipos nodulantes IAC-14, IAC-15 e da linhagem isogênica nodulante D71.9230 permaneceram sempre verde escuras nos tratamentos inoculados, o que indica ter havido fixação de nitrogênio.

Ao contrário do acúmulo de matéria seca da parte aérea, o das raízes não foi um bom parâmetro para discriminar o crescimento das plantas em função dos tratamentos, devido à menor amplitude observada (Tabela 1). Assim, aos $30 \mathrm{DAE}$, somente o cultivar IAC-15 apresentou-se significativamente inferior a todos os outros genótipos, e essa diferença desapareceu na avaliação feita aos 48 dias. Na época de pleno florescimento, examinando as raízes, foi observada abundante nodulação nos tratamentos nodulantes e que haviam sido inoculados (IAC-14, IAC15 e D71.9230). Por outro lado, nos tratamentos D71.92.31 (com e sem inoculação) e D71.9231 (com inoculação $+100 \mathrm{~kg} /$ ha de N) foi observa- 
Tabela 1. Efeito nas três épocas de colheita sobre a produção de matéria seca da parte aérea e das raízes de três plantas de genótipos de soja e isolinhas (nodulante e não) em função da inoculação com rizóbio e aplicação de $\mathrm{N}$ mineral.

\begin{tabular}{|c|c|c|c|c|c|c|}
\hline \multirow[b]{2}{*}{ Genótipos } & \multicolumn{3}{|c|}{ Época de Colheita } & \multicolumn{3}{|c|}{ Regressão } \\
\hline & $30 \mathrm{DAE}^{2}$ & $48 \mathrm{DAE}$ & $56 \mathrm{DAE}$ & Linear & Quadr. & $\mathrm{R}^{2}$ \\
\hline \multicolumn{7}{|c|}{ M. seca parte aérea (g)3plants } \\
\hline IAC-14 (nod.) inoculado & $7,44 c^{1}$ & $24,58 \mathrm{a}$ & $52,13 \mathrm{bc}$ & • & - & 0,98 \\
\hline IAC-15 (nod.) inoculado & $7,82 \mathrm{bc}$ & 25,36 a & $54,45 a b c$ & $\cdot$ & ns & 0,96 \\
\hline D71.9230 (nod.) inoculado & $9,22 \mathrm{a}$ & 20,71 a & $57,16 a b$ & $\cdot$ & - & 0,96 \\
\hline D71.9231 (não nod.) inoculado & $9,27 \mathrm{a}$ & 24,06 a & $47,84 \mathrm{c}$ & $\cdot$ & $\cdot$ & 0,98 \\
\hline D71.9231 (não nod.) sem inoc & $8,48 a b$ & $23,76 \mathrm{a}$ & $48,30 \mathrm{c}$ & $\cdot$ & $\cdot$ & 0,97 \\
\hline $\begin{array}{l}\text { D71.9231 (nāo nod.) sem inoc }+100 \\
\mathrm{~kg} / \mathrm{ha} \mathrm{N}\end{array}$ & $8,58 a b$ & $21,63 \mathrm{a}$ & 60,39 a & • & $\cdot$ & 0,97 \\
\hline $\mathrm{CV}$ & \multicolumn{3}{|c|}{$13,71 \%$} & & & \\
\hline \multicolumn{7}{|c|}{ M. seca da raiz (g) de 3 plantas } \\
\hline IAC-14 (nod.) inoculado & $2,26 \mathrm{a}$ & $11,99 \mathrm{a}$ & $13,73 \mathrm{a}$ & $\cdot$ & $\cdot$ & 0,99 \\
\hline IAC-15 (nod.) inoculado & $1,71 \mathrm{~b}$ & $12,23 \mathrm{a}$ & 13,22 a & $\cdot$ & $\cdot$ & 0,95 \\
\hline D71.9230 (nod.) inoculado & $2,69 \mathrm{a}$ & $9,78 \mathrm{a}$ & $13,83 \mathrm{a}$ & $\cdot$ & Ns & 0,95 \\
\hline D71.9231 (nāo nod.) inoculado & $2,47 a$ & $10,12 \mathrm{a}$ & $11,25 b$ & $\cdot$ & $\cdot$ & 0,97 \\
\hline D71.9231 (não nod.) sem inoc & $2,25 \mathrm{a}$ & $10,45 \mathrm{a}$ & $11,29 b$ & $\cdot$ & $\cdot$ & 0,97 \\
\hline $\begin{array}{l}\text { D71.9231 (não nod.) sem inoc + } 100 \\
\mathrm{~kg} / \mathrm{ha} \mathrm{N}\end{array}$ & $2,24 \mathrm{a}$ & $9,28 \mathrm{a}$ & $12,63 a b$ & • & - & 0,97 \\
\hline $\mathrm{CV}$ & & $11,85 \%$ & & & & \\
\hline
\end{tabular}

'Médias seguidas de letras distintas nas colunas diferem signifcativamente entre si pelo teste de Duncan a 5\%

${ }^{2} \mathrm{DAE}=$ dias após emergência 
da ausência de nódulos, conforme esperado. Aos 56 DAE, nos tratamentos nodulantes, a massa seca das raízes foi sempre superior à da isolinha não-nodulante D71.9231 (com ou sem inoculação ) e à da D71.92.31(com inoculação) $+100 \mathrm{~kg} / \mathrm{ha}$ de $\mathrm{N}$, o que pode ser atribuido à presença dos nódulos nas raízes das plantas nodulantes. Os tratamentos de inoculação (com ou sem) na isolinha não nodulante D71.9231 não proporcionaram diferenças sobre as variáveis de acúmulo de matéria seca, tanto da parte aérea como das raízes.

Observa-se que o aumento dos teores de $\mathrm{N}$-total no intervalo de 30 a 56 DAE para todos os tratamentos, ocorreu de forma linear e/ou quadrática (Tabela 2). Na avaliação feita aos $30 \mathrm{DAE}$ na parte aérea, não se observaram diferenças significativas entre os tratamentos, enquanto que nas outras duas épocas de colheita, as diferenças foram notáveis. Observou-se que os nodulantes acumularam substancialmente muito mais $\mathrm{N}$, principalmente aos $56 \mathrm{DAE}$, em relação à isolinha não nodulante. Neste material, devido à quase inoperância do sistema em fixar $\mathrm{N}$ atmosférico, a aplicação de $\mathrm{N}$ mineral aumentou a quantidade de $\mathrm{N}$ total absorvido na parte aérea das plantas, sem, no entanto, atingir a magnitude dos outros genótipos com capacidade de nodular. Para que as isolinhas não-nodulantes igualassem e ultrapassassem as nodulantes, talvez fosse necessário o uso de doses mais elevadas de N. Não houve variação significativa no teor de $\mathrm{N}$ do sistema radicular dos isolinhas e dos genótipos avaliados.

Na Tabela 3, observa-se que, do total de $\mathrm{N}$ absorvido, as quantidades fixadas do ar pelos cultivares IAC-14, IAC-15 e a linhagem nodulante D71.9230 foram de 62, 62 e 64\%, respectivamente, enquanto aquelas absorvidas do solo foram de 38,38 e $36 \%$. Por outro lado, a linhagem não-nodulante D71.9231 retirou apenas $11 \%$ de nitrogênio do fertilizante, enquanto $89 \%$ foram aproveitado do solo. Conforme Jenkinson, citado por Hauck e Bremner (1976), haveria um efeito chamado "Priming": ou "Added nitrogen interaction": a aplicação de N mineral como fertilizante estimularia a mineralização da fração orgânica do elemento presente no solo. Por esta razão é que apenas $11 \%$ de nitrogênio foi proveniente do fertilizante enquanto que a maior quantidade ( $89 \%$ ) 
Tabela 2. Efeito nas três épocas de colheita sobre o $\mathrm{N}$ total acumulado na matéria seca da parte aérea e das raízes dos genótipos de soja (nodulante ou não) em função da inoculação com rizóbio e aplicação de $\mathrm{N}$ mineral.

\begin{tabular}{|c|c|c|c|c|c|c|}
\hline \multirow{2}{*}{ Genótipos } & \multicolumn{3}{|c|}{ Época de Colheita } & \multicolumn{3}{|c|}{ Regressão } \\
\hline & $30 \mathrm{DAE}$ & $48 \mathrm{DAE}$ & 56 DAE & Linear & Quadr. & $R^{2}$ \\
\hline \multicolumn{7}{|c|}{$\mathrm{N}$ total da parte aérea (mg) 3plts. } \\
\hline IAC-14 (nod.) inoculado & $250 \mathrm{a}$ & $571 \mathrm{a}$ & $1296 a$ & $\cdot$ & ns & 0,86 \\
\hline IAC-15 (nod.) inoculado & $240 \mathrm{a}$ & $528 \mathrm{ab}$ & $1255 a$ & $\cdot$ & ns & 0,86 \\
\hline D71.9230 (nodulante) inoculado & 252 a & $384 a b$ & $1230 a$ & $\cdot$ & $*$ & 0,89 \\
\hline D71.9231 (nāo nodulante) inoculado & $262 \mathrm{a}$ & $350 \mathrm{~b}$ & $421 \mathrm{~b}$ & $\cdot$ & ns & 0,73 \\
\hline D71.9231 (não nodulante) sem inoc & $252 a$ & $360 \mathrm{~b}$ & $421 \mathrm{~b}$ & * & * & 0,64 \\
\hline $\begin{array}{l}\text { D71.9231 (não nododulante) sem inoc + } \\
100 \mathrm{~kg} / \mathrm{ha} \mathrm{N}\end{array}$ & $276 \mathrm{a}$ & $479 a b$ & $721 b$ & $\cdot$ & ns & 0,76 \\
\hline $\mathrm{CV}$ & \multicolumn{3}{|c|}{$27,66 \%$} & & & \\
\hline \multicolumn{7}{|c|}{-..-.- $\mathrm{N}$ total na raiz $(\mathrm{mg})$} \\
\hline IAC-14 (nodulante) inoculado & $47 a$ & 189 a & 257 a & $\cdot$ & ns & 0,91 \\
\hline IAC-15 (nodulante) inoculado & $33 \mathrm{~b}$ & $192 \mathrm{a}$ & $204 \mathrm{~b}$ & $\cdot$ & $\cdot$ & 0,88 \\
\hline D71.9230 (nodulante) inoculado & $55 \mathrm{a}$ & $151 \mathrm{a}$ & 271 a & $\cdot$ & ns & 0,83 \\
\hline D71.9231 (não nodulante) inoculado & $48 a$ & $124 a$ & $126 \mathrm{c}$ & $\cdot$ & $\cdot$ & 0,88 \\
\hline D71.9231 (não nodulante) sem inoc & $45 a$ & $126 \mathrm{a}$ & $119 \mathrm{c}$ & $\cdot$ & $\cdot$ & 0,90 \\
\hline $\begin{array}{c}\text { D71.9231 (não nodulante) sem inoc + } \\
100 \mathrm{~kg} / \mathrm{ha} \mathrm{N}\end{array}$ & $47 a$ & $127 a$ & $145 c$ & - & ns & 0,70 \\
\hline CV & & $24,23 \%$ & & & & \\
\hline
\end{tabular}

Médias seguidas de letras distintas nas colunas diferem signifcativamente entre si pelo teste de Duncan a 5\%

$\mathrm{DAE}=$ dias após emergência 
Tabela 3. Quantidade de $\mathrm{N}$ total, fixado e retirado do solo, em mg e \% na parte aérea e raízes dos cultivares IAC14, IAC-15 e as isolinhas isogênicas D72.9230 e D71.9231+100kg/ha de $\mathrm{N}$ total fixado (parte aérea + raízes) e retirado do solo em kg/ha.

\begin{tabular}{|c|c|c|c|c|c|c|c|c|c|c|c|}
\hline \multirow[t]{3}{*}{ Cultivar } & \multicolumn{2}{|c|}{$\mathrm{N}$ total } & \multicolumn{2}{|c|}{$\begin{array}{l}\text { Quantidade de } N \\
\text { fixada por } 3 \text { plantas }\end{array}$} & \multicolumn{2}{|c|}{$\begin{array}{l}\text { Quantidade de } \\
\text { de } N \text { retirada } \\
\text { do solo }\end{array}$} & \multicolumn{2}{|c|}{ Total fixado } & \multicolumn{2}{|c|}{$\begin{array}{l}\text { Tolal retirado } \\
\text { do solo }\end{array}$} & \multirow[t]{2}{*}{$\begin{array}{l}\text { Total } \\
\text { retirado }\end{array}$} \\
\hline & \multicolumn{10}{|c|}{ Parle aérea } & \\
\hline & $\mathrm{mg}$ & $\%$ & $\mathrm{mg}$ & $\%$ & $\mathrm{mg}$ & $\%$ & $\mathrm{~kg} / \mathrm{ha}$ & $\%$ & $\mathrm{~kg} / \mathrm{ha}$ & $\%$ & $\mathrm{~kg} / \mathrm{ha}$ \\
\hline IAC-14 (nodulante) & 1296 & 100 & 804 & 62 & 492 & 38 & 75,6 & 62 & 53,8 & 38 & 129,4 \\
\hline |AC-15 (nodulante) & 1255 & 100 & 779 & 62 & 476 & 38 & 73,7 & 62 & 52,5 & 38 & 126.2 \\
\hline D71.9230 (nodulanie) & 1230 & 100 & 787 & 64 & 443 & 36 & 80,5 & 64 & 44,6 & 36 & 125,1 \\
\hline \multirow[t]{2}{*}{$D 71.9231+100 \mathrm{~g} / \mathrm{ha} \mathrm{N}$} & 721 & 100 & 79 & $11^{1}$ & 642 & 89 & 18,3 & $11^{t}$ & 64,20 & 89 & 82,5 \\
\hline & & Raize & & & & & & & & & \\
\hline IAC-14 (nodulante) & 257 & 100 & 103 & 58 & 154 & 42 & $\cdot \cdot$ & - & - & & • \\
\hline IAC-15 (nodulante) & 204 & $100^{\circ}$ & 106 & 52 & 98 & 48 & $\therefore$ & - & • & & - \\
\hline D71.9230 (nodulante) & 271 & 100 & 179 & 66 & 92 & 34 & $\cdot \cdot$ & - & - & & - \\
\hline $071.9231+100 \mathrm{~g} / \mathrm{ha} \mathrm{N}$ & 145 & 100 & 179 & 66 & 92 & 34 & $\cdot \cdot$ & . & - & & $\cdot$ \\
\hline
\end{tabular}

'Retirada do fertilizante

foi retirada do solo. Tendências semelhantes foram observadas nas raízes e as quantidades retiradas do solo foram semelhantes. A isolinha D71.9230 (nodulante) fixou um pouco mais de $\mathrm{N}$ do ar do que os cultivares IAC-14 e IAC-15. Por outro lado, a isolinha D.71.9231 (não nodulante) mostrou que $100 \mathrm{~kg} /$ ha de $\mathrm{N}$ aplicado não foram suficientes para suprir a necessidade das plantas que apresentaram sintomas de deficiência de $\mathrm{N}$ na parte aérea. Os cultivares IAC-14, IAC-15 e isolinha D71.9230 retiraram no total 129,126 e $125 \mathrm{~kg} / \mathrm{ha}$ ( $\mathrm{N}$ fixado $+\mathrm{N}$ solo), enquanto que a isolinha D71.9331 retirou apenas $83 \mathrm{~kg} / \mathrm{ha}$ de $\mathrm{N}$. 


\section{REFERÊNCIAS BIBLIOGRÁFICAS}

ALLEN, O.N. \& I.L. BALDWIN, 1954. Rhizobia-Legume Relationships, Soil Sci., 78:415-427.

ALLOS, H.F. \& W.V. BARTHOLOMEW, 1959. Replacement of Symbiotic Fixation by Available Nitrogen. Soil Sci., 87:61-66. BREMNER, J.M. Total Nitrogen. In: BLACK, C.A., ed., 1965a. Methods of Soil Analysis. Part 2. Madison, Am. Soc. Agron., p.1149-1178. BREMNER, J.M., 1965b. Isotope Rationanalysis of Nitrogen in Nitrogen-15. Tracer Investigation. Part 2. Madison, Am. Soc. Agron., p.1256-1286.

BREMNER, J.M. \& C.S. MULVANEY, 1982. Total Nitrogen. In:BLACK C.A. ed. Methods of Soil Analysis. 2 ed. Madison, Am. Soc. Agron., p.1256-1286.(Agronomy Series of Monography No9).

FRIED, M. \& V. MIDDLEBOE, 1977. Measurement of Amount of

Nitrogen Fixed by a Legume Crop. Plant and Soil, 47:713-715.

HARPER, J.E., 1974. Soil and Symbiotic Nitrogen Requirements for Optimum Soybean Production. Crop Sci., 14:255-260.

HAUCK, R.D. \& J.M. BREMNER, 1976. Use of Tracers for Soil and

Fertilizer Nitrogen Research. Advances in Agronomy, New York, 28:219-266.

HINSON, K., 1974. Nitrogen Fertilization of Soybeans (Glycine max (L.) Merrill) in Peninsular Florida. Soil and Crop Sci. Soc. of Florida, 24:97-101.

HUNGRIA, M; R.J. CAMPO; M.A.T. VARGAS; D.S. ANDRADE. Fixação Biológica de Nitrogênio em Leguminosas de Grãos. In XXII Reunião Bras. Fertilidade do solo e Nutrição de Plantas em Caxambu, Resumo p 29-30, 1998.

MASCARENHAS, H.A.A.; S. MIYASAKA; G. SORDI \& E.S. LOPES, 1967. Efeito da Inoculação das Sementes com Rhizobium e da Subsequente "Peletização" com Pasta de Carbonato de Cálcio na Ausência e na Presença da Calagem e da Adubação Nitrogenada. Bragantia 26: 143-153. 
MASCARENHAS, H.A.A.; E.A. BULISANI; O.C. BATAGLIA \& S.M.P. FALIVENE, 1984. Produção, Absorção e Extração de Nutrientes por Linhagem de Soja que Nodula e que Não Nodula. I. Efeito do nitrogênio mineral e da inoculação. Bragantia, Campinas, 43(2):397404.

McAULIFFE, C.; D.S. CHAMBLEE; H. URIBE-ARANGO \& W.W. WOODHOUSE, 1958. Influence of Inorganic Nitrogen Fixation by Legumes as Revealed by ${ }^{15}$ N. Agron. J., 50: 334-347.

NORMAN, A.G., 1944. The Nitrogen Nutrition of Soybeans. I The Nitrogen Nutrition of Soybeans. I. Effect of Inoculation and Nitrogen Fertilizer on the Yield and Compositon of Beans on Marchall Silt Loam. Soil Sci. Soc. Am. Proc. 8:226-228.

NORMAN, A.G. \& L.O. KRAMPITZ, 1945. The Nitrogen Nutrition of

Soybeans. II Effect of Available Soil Nitrogen on the Growth and

Nitrogen Fixation. Soil Sci. Soc.of Am., Proc., 10:191-196.

NOVO, M.C.S.S.; R.T. TANAKA; H.A.A. MASCARENHAS;

J.C.V.N.A. PEREIRA; N. BORTOLETTO; P.B. GALLO \& A.A.T.

VARGAS, 1997. Influencia do Nitrogênio e do Potássio no Crescimento e no Rendimento da Soja Cultivada no Inverno. Pesq. Agropec. Bras., Brasília, 32: 33-41.

TRIVELIN, P.C.O.; E. SALATI \& E. MATSUI., 1973. Preparo de Amostras para Análise de ${ }^{15} \mathrm{~N}$ por Espectrometria de Massa. Piracicaba, CENA, 41p. (Boletim Tec. 2).

UMBREIT, W.W.\& E.B. FRED, 1936. The Comparative Efficiency of

Free and Combined Nitrogen to the Nutriton of Soybeans. J. Am.

Soc. Agron. 28:548-555.

WEBER, C.R., 1966a. Nodulation and Non-Nodulation Soybean Isolines.

I Agronomic and Chemical Attributes. Agron. J., 58:43-46.

WEBER, C.R., 1966b. Nodulating and Non-Nodulating Isolines. II.

Response to Applied Nitrogen and Modified Soil Conditions. Agron. J., 58:46-48. 